\title{
Causality in Non-Hausdorff Space-Times
}

\author{
P. HAJICEK \\ Institute for Theoretical Physics, University of Berne
}

Received January 1, 1971

\begin{abstract}
Some general properties of completely separable, non-Hausdorff manifolds are studied and the notion of a non-Hausdorff space-time is introduced. It is shown that such a space-time must, under very general conditions, display a kind of causal anomaly.
\end{abstract}

\section{Introduction}

This paper is devoted to the study of a class of singularities exhibited by solutions of Einstein's equations. In the recent literature, several attempts of this sort appeared especially after the singularity theorems had been proved (e.g., in $[1,2])$ stating that some pathological features must be present without much more information as to the extent of this pathology [3]. In some papers, such as [4, 5], various causal anomalies, geodesic incompleteness, anomalous sources, etc., in a concrete family of solutions are described. In other papers, one of these properties is investigated quite generally, for example the causal geodesic incompleteness [6] or incompleteness [7]. The problem to be solved here is to find general behaviour of those solutions whose maximal analytic extension is non-Hausdorff. At the first glance, these spaces seemed to be very strange containing bifurcate geodesics, curves with more than one endpoint, etc., [6]. Then, it has been shown that no such strangeness is present in a non-Hausdorff extension of the Taub-NUT space $[8,9]$. Nevertheless, we shall see that all such space-times must be weakly acausal (Theorem 4).

\section{Structure of Non-Hausdorff Manifolds}

Non-Hausdorff manifolds are defined for instance in [10], p. 2. We consider here only a special case of them: the completely separable ones. First some symbolics. A non-Hausdorff, completely separable, $n$-dimensional, differentiable $\left(C^{k}\right)$ manifold will be shortly said $Y$-manifold. In a $Y$-manifold $W$, there are at least two points $p$ and $q$ for which we find no two open disjoint sets $A$ and $B$ such that $p \in A$ and $q \in B$. This relation of points $p$ and $q$ will be written $p \curlyvee q$. If $M$ and $N$ are some subsets of $W$, then $Y_{M}^{N}$ denotes the set of all points $x \in M$ for which there is $y \in N$ 
such that $x y y . W$ as an index at $Y$ will be usually omitted. The closure (boundary) of a set $M$ in a topological subspace $N$ of $W$ will be denoted by $\bar{M}^{N}\left(\dot{M}^{N}\right)$; similarly, $W$ as an index here will not appear. The next convention to make is the following: $\{p\}$ denotes the set whose element is $p ;\left\{p_{i}\right\}$ is a sequence of points $p_{i} ;\{C\}$ is the set of all points lying on the curve $C$, etc. In what follows, the notion of open submanifold will only be used for connected open submanifolds. We introduce a sum of open submanifolds $U, V$ of a manifold $W$ : if $N \cap V \neq \emptyset$, then the set $U \cup V$ is open and connected; equipped by the differentiable structure induced by that of $W$ it is an open submanifold of $W$ and will be denoted by $U+V$.

Every point of a $Y$-manifold $W$ lies in an open submanifold which is Hausdorff, because $W$ is locally homeomorph to $R^{n}$. We define

Definition 1. An open submanifold $V$ of a $Y$-manifold $W$ is called $H$-submanifold, if

1) $V$ is Hausdorff,

2) $V$ is not a proper subset of any other open Hausdorff submanifold of $W$.

Any $H$-submanifold of $W$ is paracompact and metrizable ([11], pp. $79,81)$.

Theorem 1. Let $W$ be a $Y$-manifold and $\mathscr{H}$ the system of all its $H$-submanifolds. Then $\mathscr{H}$ is an open covering of $W$.

Proof. Let $\Omega$ denote the system of all open Hausdorff submanifolds of $W$. If $U \in \Omega, V \in \Omega$ and $U$ is a proper subset of $V$, we write $U<V$. For arbitrary $U, V, X$ from $\Omega$ it holds

1) if $U<V$, then $V<U$ is false,

2) if $U<V, V<X$, then $U<X$.

Therefore, $\Omega$ is partially-ordered by $<$. Let $p \in W$ and $U \in \Omega$ such that $p \in U .\{U\}$ is a non-empty subset of $\Omega$ which is simply-ordered by $<$. According to Maximal Principle ([11], p. 25), there is at least one simplyordered subset $\Pi \in \Omega$ which contains $U$ and is not a proper subset of any other subset of $\Omega$ with these properties. Denote $P=\sum_{U \in \Pi} U$. The sum makes sense, because every two elements of $\Pi$ have a non-empty intersection. $P$ is an open submanifold of $W$ and must be Hausdorff: let $x \in P, y \in P$ be two arbitrary points; then, there are $M \in \Pi$ and $N \in \Pi$ such that $x \in M, y \in N$. But we have either $M<N$ or $N<M$, so that both points $x$ and $y$ lie in an open Hausdorff submanifold of $W$ and $x y y$ must be false. By construction, $P$ is maximal and $p \in P$, q.e.d. ${ }^{1}$

Theorem 2. Let $V$ be an open submanifold of a $Y$-manifold $W$. Necessary and sufficient condition for $V$ to be an $H$-submanifold of $W$ is the relation $\dot{V}=\overline{Y^{V}}$.

1 The relation" $Y$ ", the Definition 1 as well as the proof of Theorem 1 can be found in $[12]$. 
Proof. 1. Let $V$ be an $H$-submanifold. If $x \in Y^{V}$, then there is $y \in V$ such that $x Y y$; therefore, $x$ is a limit point of $V$ not lying in $V$ and we have $Y^{V} \subset \dot{V}$. Let $x \in \dot{V}$ have a neighbourhood $O$ such that $O \cap Y^{V}=\emptyset$. Then, there is an open Hausdorff submanifold $U$ satisfying $x \in U \subset O$. $U \cap V \neq \emptyset$, so the sum $V^{\prime}=V+U$ makes sense and $V<V^{\prime}$, because $x \notin V$. Next, $V^{\prime}$ is Hausdorff: suppose that there are two points $p$ and $q$ in $V^{\prime}$ with $p \curlyvee q$. $p$ and $q$ cannot then both lie in $V$ or both lie in $U$; but it is impossible, that, e.g., $p \in V$ and $q \in U$, too, because $U$ does not contain any point of $Y^{V}$. Thus, $V$ would not be maximal, contrary to the hypothesis, hence $x$ must be a limit point of $Y^{V}$, or $\dot{V} \subset \overline{Y^{V}}$. But $\dot{V}$ is closed, so that from $Y^{V} \subset \dot{V} \subset \overline{Y^{V}}$ it follows $\dot{V}=\overline{Y^{V}}$.

2. Let $V$ be an open submanifold of $W$ such that $\dot{V}=Y^{V}$. Then $Y^{V} \subset \dot{V}$ and $V$ must be Hausdorff or else there would be a point $x \in V$, $x \in Y^{V}$, and this implies $V \cap \dot{V} \neq \emptyset$. Let $V^{\prime}$ be an open submanifold of $W$ such that $V<V^{\prime}$. Then the boundary of $V$ in $V^{\prime}$ is not empty; it is the set $\dot{V} \cap V^{\prime}$. Choose $p \in \dot{V} \cap V^{\prime}$; according to $\dot{V}=\overline{Y^{V}}$, in every neighbourhood of $p$ there are points of $Y^{V}$. But we can find a neighborhood of $p$ lying entirely in $V^{\prime}$. Hence, $V^{\prime}$ cannot be Hausdorff and $V$ is maximal, q.e.d.

It can easily be seen that every point of $Y$ lies on the boundary of some $H$-submanifold. Another trivial result is the equivalence of the following two claims

1) $p Y q$.

2) There is a sequence of points converging to both $p$ and $q$.

A more difficult question is the following: let $p Y q$ and $\left\{p_{i}\right\}$ be a sequence of points converging to $p$. What conditions must be imposed on $\left\{p_{i}\right\}$, in order that it converges also to $q$ ? Trying to answer this, we learn about importance of the $H$-manifolds boundary shape. Therefore, we are led to the abbreviation:

Definition 2. Let $W$ be a $Y$-manifold and $V$ one of its $H$-submanifolds. Then $\dot{V}$ is called a $Y$-boundary in $W$.

We need the following

Definition 3. Let $W$ be a manifold and $O$ an open subset in $W$. We say that $\dot{O}$ is simple at a point $p \in \dot{O}$, if for every open set $U$ containing $p$ there is an open set $U^{\prime}$ containing $p$ and contained in $U$ such that $U^{\prime} \cap O$ is connected.

Theorem 3. Let $W$ be a $Y$-manifold, and $U$ and $V$ its $H$-submanifolds such that $Z=U \cap V \neq \emptyset$, and $p \in Y_{U}^{V}$. Suppose $\dot{Z}^{U}$ is simple at $p$. Then the following claims are equivalent: in $V$.

1) Every sequence of points $p_{i} \in Z$ converging to $p$ has a limit point

2) $Y_{V}^{p}$ is compact. 
Proof. 1) $Y_{V}^{p}$ is non-empty, because $p \in Y_{U}^{V}$. We assume that $Y_{V}^{p}$ is not compact. Then there is a sequence of points $q_{i} \in Y_{V}^{p}$ which has no limit point in $Y_{V}^{p}: V$ is metrizable, therefore the compactness is equivalent to the countable compactness. Let us denote by $\varrho$ a possible metrics on $V$, let $\left\{K_{i}\right\}$ be a sequence of open spheres $K_{i}$ with the radius $1 / i$ as measured by $\varrho$ and centre $q_{i}$, and $\left\{U_{i}\right\}$ be a sequence of neighbourhoods of $p$ converging to $p$. Because of $p \curlyvee q_{i}, K_{i} \cap U_{i}$ is non-empty, and we can choose a point $p_{i} \in K_{i} \cap U_{i}$. The sequence $\left\{p_{i}\right\}$ converges to $p$ by construction. Suppose that it has a limit point $q \in V$. Let $O_{p}$ be a neighbourhood of $p$ and $O_{q}$ a neighbourhood of $q$. There is a spherical neighbourhood $K_{q}^{1}$ of centre $q$ and radius $r$ such that $K_{q}^{1} \subset O_{q}$. Choose $K_{q}^{2}$ with centre $q$ and radius $r / 2$. As $q$ is a limit point of $\left\{p_{i}\right\}$, there is a subsequence $\left\{p_{i_{k}}\right\}$ such that for every $k>N_{1}$ we have $p_{i_{k}} \in K_{q}^{2}$. Next, there is $N_{2}$ such that $i_{k}>2 / r$ for every $k>N_{2}$. But then the point $p_{i_{N_{1}+N_{2}}}$ lies in $K_{q}^{2}$ and $\varrho\left(q_{i_{N_{1}+N_{2}}}\right.$, $p_{i_{N_{1}+N_{2}}}$ ) $<r / 2$, hence $q_{i_{N_{1}+N_{2}}} \in O_{q}$ and $O_{q}$ is a neighbourhood of $q_{i_{N_{1}+N_{2}}}$. But then $O_{q} \cap O_{p} \neq \emptyset$ and because they were arbitrary, we have $p \curlyvee q$. On the other hand, $q$ is a limit point of $\left\{q_{i}\right\}$, contrary to the hypothesis that $\left\{q_{i}\right\}$ has no limit point in $Y_{V}^{p}$.

2) Now, let $Y_{V}^{p}$ be compact. Suppose that every point $x \in Y_{V}^{p}$ has a neighbourhood $O_{x} \subset V$ whose closure $\bar{O}_{x}^{V}$ is compact and contains no point of $\left\{p_{i}\right\}$. We can choose some finite number of $O_{x}$ covering $Y_{V}^{p}$, denote these by $O_{i}, i=1, \ldots, N$. If $O=\bigcup_{i=1}^{N} O_{i}$, then $\bar{O}^{V}$ is compact, so that $\dot{O}^{V}$ is compact, too, because $V$ is Hausdorff. Next, choose a sequence $\left\{U_{i}\right\}$ of neighbourhoods of $p$ in $U$ converging to $p$ such that $p_{i} \in U_{i}$ and $Z \cap U_{i}$ is connected for every $i$. $U_{i} \cap Z$ is also locally connected and locally compact, because it is a manifold. As a Hausdorff submanifold it is metrizable. All these properties imply that $U_{i} \cap Z$ is arcwise connected ([11], p. 115). Let $q$ be a point of $Y_{V}^{p}$ and $\left\{V_{i}\right\}$ a sequence of neighbourhoods of $q$ converging to $q$. Choose $q_{i} \in U_{i} \cap V_{i}$; then $q_{i} \in U_{i} \cap Z$ and there is an arc $\lambda_{i}$ with endpoints $p_{i}$ and $q_{i}$ lying in $U_{i} \cap Z$. We find $N^{\prime}$ such that $q_{i} \in O$ for every $i>N^{\prime}$. Therefore, $\lambda_{i}$ must cut $\dot{O}^{V}$ for every $i>N^{\prime}$ in a point, say, $r_{i}$. Let $r$ be a limit point of the sequence $\left\{r_{i}\right\}$ on $\dot{O}^{V}$. This sequence converges to $p$ because of $\left\{\lambda_{i}\right\} \subset U_{i}$. But then $r \nmid p$, since $r \in V$, contrary to $\dot{O}^{V} \cap Y_{V}^{p}=\emptyset$.

If $\dot{Z}^{U}$ is not simple at $p, 1$ ) still implies 2), but the converse is not necessarily true.

Example. Let $M^{\prime}$ be the two-dimensional Minkowski space-time without the origin and $\tilde{M}^{\prime}$ be the universal covering space of $M^{\prime}$. The coordinates on $M^{\prime}$ they are $x, y ; d s^{2}=d x^{2}-d y^{2}$; on $\tilde{M}^{\prime}$ are $\xi, \eta, n$, where $n$ is an integer (because the first homotopy group of $M^{\prime}$ is isomorphic to $Z$ ); $d s^{2}=d \xi^{2}-d \eta^{2}$. In $M^{\prime}$, let $N^{\prime}$ be defined by $N^{\prime}=M^{\prime}$ 
$-\{p \mid y(p)=0, x(p)>0\}$, and in $\tilde{M}^{\prime}$, let $\tilde{N}^{\prime}$ be defined by

$$
\tilde{N}^{\prime}=\left\{p \in M^{\prime} \mid n(p)=0\right\}-\left\{p \in M^{\prime} \mid \eta(p)=0, \xi(p)>0\right\} .
$$

Then the map $\varphi: N^{\prime} \rightarrow \tilde{N}^{\prime}$ defined by

$$
n=0, \quad \xi=x, \quad \eta=y
$$

is one-to-one and isometry. Identifying the spaces $M^{\prime}$ and $\tilde{M}^{\prime}$ along the open sets $N^{\prime}$ and $\tilde{N}^{\prime}$ according to (1), we get a two-dimensional $Y$-manifold $M . M^{\prime}$ and $\tilde{M}^{\prime}$ are the only $H$-submanifolds of $M$. Boundary of $M^{\prime}$ in $M$ consists of two segments $n=0, \xi>0, \eta=0$, and $n=1, \xi>0, \eta=0$, boundary of $\tilde{M}^{\prime}$ in $M$ is the segment $x>0, y=0$; the latter is not simple in any of its points. Now, delete the point $p_{2}^{\prime}$ defined by $\xi\left(p_{2}^{\prime}\right)=1$, $\eta\left(p_{2}^{\prime}\right)=0, n\left(p_{2}^{\prime}\right)=1$ from $M^{\prime}$. The sequence of points with coordinates $x_{i}=1, y_{i}=-1 / i$ converges in $M^{\prime}$ to $p_{1}$ defined by $x\left(p_{1}\right)=1, y\left(p_{1}\right)=0$, but does not converge in $\tilde{M}^{\prime}-\left\{p_{2}^{\prime}\right\}$, though the set $Y_{\tilde{M}^{\prime}-\left\{p^{\prime}{ }^{2}\right\}}^{p_{1}}$ is compact: it contains just one point $p_{1}^{\prime}$ defined by $\xi\left(p_{1}^{\prime}\right)=1, \eta\left(p_{1}^{\prime}\right)=0, n\left(p_{1}^{\prime}\right)=0$.

\section{Causality in $Y$-space-times}

In this section, we specialize our analysis to four-dimensional $Y$-manifolds of differentiability class $\left(C^{2}, C^{4}\right.$ piecewise $)$ bearing a Lorentzian metric of signature -2 and differentiability class $\left(C^{1}, C^{3}\right.$ piecewise), which will be shortly called $Y$-space-times.

A dominant role will be played by curves. For the sake of convenience, we shall understand here under a curve $C$ a smooth map $C$ of one of the following four types of interval into a $Y$-manifold $W:[a, b]$, $(a, b),[a, b),(a, b]$, where $a, b$ are reals. The curve is time-like (causal), if its tangent vector at every point as well as its limits as $t \rightarrow a$ or $t \rightarrow b$ is time-like (time-like or light-like).

Definition 4. Bifurcate curve $\left(C_{1}, C_{2}, g\right)$ in a $Y$-manifold $W$ is a pair of curves $C_{1}:[a, b] \rightarrow W, C_{2}:[a, b] \rightarrow W$, and a number $g$ such that $a<g \leqq b, C_{1}(t)=C_{2}(t)$ for $a \leqq t<g$ and $C_{1}([g, b]) \cap C_{2}([g, b])=\emptyset$.

It is easily seen that such curves can only exist in a non-Hausdorff space. Then, if we have some system of ordinary differential equations which has locally a unique solution for a given set of initial data (e.g., the geodesic equations with initial point and velocity, as well as other classical dynamical equations), it is immediate that this system cannot have two different solutions for this data, unless these solutions form a bifurcate curve. Therefore, in view of the classical causality conception coinciding with determinism it is sensible to rule out the bifurcate curves. Our main result is 
Theorem 4. Let $M$ be a $Y$-space-time whose $Y$-boundaries are all three-dimensional hypersurfaces (not necessarily smooth). Then the following two requirements are not compatible:

1) There are no time-like bifurcate curves with bounded acceleration in $M$.

2) $M$ is strongly causal.

To prove the Theorem 4, we need the following Lemmas.

Lemma 1. There is a bifurcate curve in a $Y$-space-time $M$, if and only if there is a pair $U, V$ of $H$-submanifolds of $M$ with a non-empty intersection $Z=U \cap V$, and a curve $C:[0,1) \rightarrow Z$ that has no endpoint in $Z$, but has an endpoint in $U$ as well as in $V$.

Proof. If $\left(C_{1}, C_{2}, g\right)$ is a bifurcate curve in $M$, then $C_{1}(g) \curlyvee C_{2}(g)$. Choose $H$-submanifolds $U$ and $V$ such that $C_{1}(g) \in U, C_{2}(g) \in V . C_{1}$ and $C_{2}$ are continuous, so there is $\delta>0$ such that $C_{1}((g-\delta, g+\delta)) \subset U$, $C_{2}((g-\delta, g+\delta)) C V$. Denote $C(t)=C_{1}(g-\delta+\delta t)=C_{2}(g-\delta+\delta t)$ for $t \in[0,1) . C$ lies in $Z$ and has endpoints $C_{1}(g)$ in $U, C_{2}(g)$ in $V$. If $C$ has an endpoint $p \in Z$, then $p \curlyvee C_{1}(g), p \curlyvee C_{2}(g)$, so that $p \notin U, p \notin V$, contrary to $p \in Z=U \cap V$.

2) The other way round is trivial.

Lemma 2. Let $V$ be a Hausdorff space-time and $C:[0,1) \rightarrow V$ a one-toone curve. Then $C$ has an endpoint $p$ in $V$ such that $p \notin\{C\}$, if and only if the set $\overline{\{C\}}-\{C\}$ consists of just one point.

Proof. Suppose that $C$ has an endpoint in $V$, so that we have a curve $C^{\prime}:[0,1] \rightarrow V, C^{\prime}(t)=C(t)$ for $t \in[0,1)$. $C^{\prime}$ is continuous, hence for every neighbourhood $O$ of $p=C^{\prime}(1)$ there is $\delta>0$ such that $C^{\prime}((1-\delta, 1]) \subset O$. Only one point of this property can exist in $V$, because $V$ is Hausdorff. Furthermore, the set $\left\{C^{\prime}\right\}$ is closed in $V . p$ cannot lie in $\{C\}$. Therefore, $\overline{\{C}^{V}-\{C\}=\{p\}$.

2) Let $\{\bar{C}\}^{V}-\{C\}=\{p\}$. Choose a sequence $\left\{O_{i}\right\}$ of neighbourhoods of $p$ converging to $p$, and in each $O_{i}$ a point $p_{i} \in O_{i} \cap\{C\}$. As $C$ is one-toone, the sequence $\left\{p_{i}\right\}$ defines uniquely the sequence $\left\{t_{i}\right\}$ such that $C\left(t_{i}\right)=p_{i} .\left\{t_{i}\right\}$ has no limit point on $[0,1)$, because $\left\{C\left(t_{i}\right)\right\}$ converges to $p$ and $V$ is Hausdorff; hence, there is a subsequence $\left\{t_{i}^{\prime}\right\}$ converging to 1 . Now, suppose that there is a neighbourhood $O$ of $p$, for which we never find $\delta>0$ such that $C((1-\delta, 1)) \subset O$. This means that for every $t_{i}^{\prime}$ there is some $s_{i}, t_{i}^{\prime}<s_{i}<1$, such that $C\left(s_{i}\right) \notin O ; \lim _{i \rightarrow \infty} s_{i}=1$. Choose a neighbourhood $B$ of $p$ that has compact closure in $V$ and $\bar{B}^{V} \subset O$. There is $N$ such that for any $i>N$ the curve $C_{i}:\left[t_{i}^{\prime}, s_{i}\right] \rightarrow V$ defined by $C_{i}(t)=C(t)$ for $t \in\left[t_{i}^{\prime}, s_{i}\right]$ has its endpoint $C_{i}\left(t_{i}^{\prime}\right)$ in $B$; its endpoint $C_{i}\left(s_{i}\right)$ cannot lie in $O$, so we can choose $q_{i} \in\left\{C_{i}\right\} \cap \dot{B}^{V}$. But $\dot{B}^{V}$ is compact and the sequence $\left\{q_{i}\right\}$ has at 
least one limit point on $\dot{B}^{V}$, say, $q$. On the other hand, $q_{i} \in\{C\}$, hence we have unique $u_{i}$ such that $C\left(u_{i}\right)=q_{i}$. By construction, $t_{i}^{\prime}<u_{i}<s_{i}$, thus, $\lim _{i \rightarrow \infty} u_{i}=1$ and $q \in\left\{C \bar{S}^{V}\right.$. But $q \notin\{C\}$ and $q \neq p$, contrary to the hypothesis.

Lemma 3. Let $V$ be a Hausdorff space-time and $C:[0,1) \rightarrow V$ be a time-like curve such that the set $\{\bar{C}\}^{V}-\{C\}$ contains more that one point. Then $V$ is not strongly causal.

Proof. Choose $p \in\left\{\bar{C}^{V}-\{C\}, q \in\left\{\bar{C}^{V}-\{C\}, p \neq q\right.\right.$, two disjoint neighbourhoods $O_{p}, O_{q}$ of $p$ and $q$, and an arbitrary neighbourhood $U_{p}$ of $p$ such that $U_{p} \subset O_{p}$. There is a point $p_{1} \in\{C\} \cap U_{p}$ and a unique number $t_{1}$ such that $C\left(t_{1}\right)=p_{1}$. If $C\left(\left(t_{1}, 1\right)\right) \cap O_{q}=\emptyset$, then $q$ would not be a limit point of $\{C\}$ not lying on $C$, and we find, therefore, $t_{2}>t_{1}$ such that $C\left(t_{2}\right) \in O_{q}$. If $C\left(\left(t_{2}, 1\right)\right) \cap U_{p}=\emptyset$, then $p \notin\left\{\overline{\{C}^{V}-\{C\}\right.$, hence there is $t_{3}>t_{2}$ such that $C\left(t_{3}\right) \in U_{p}$. But then the time-like curve $C$ goes through $U_{p}$ more that once, exactly what is forbidden by the condition of strong causality.

Proof of the Theorem 4: Suppose that $Y$-space-time $M$ satisfies the condition of Theorem 4 and the requirements 1) and 2). $M$ has at least two $H$-submanifolds $U$ and $V$ with non-empty intersection $Z$. Choose a smooth time-like curve $C:[0,2] \rightarrow U^{2}$ with bounded acceleration such that $\left.C\right|_{(0,1)}$ lies in $Z$ and $C(1)=p \in \dot{Z}$. Let $U_{p}$ be a coordinate neighbourhood of $p$ with coordinates $x^{0}, x^{1}, x^{2}, x^{3}$. Next, choose a three-parameter congruence of time-like curves $\Gamma\left(t ; u^{1}, u^{2}, u^{3}\right)$ in $U$ satisfying:

1) There is $\varepsilon>0$ such that

$$
\frac{\partial\left(x^{0}, x^{1}, x^{2}, x^{3}\right)}{\partial\left(t, u^{1}, u^{2}, u^{3}\right)} \neq 0
$$

for $0<t<2$ and $\left(u^{1}\right)^{2}+\left(u^{2}\right)^{2}+\left(u^{3}\right)^{2}<\varepsilon^{2}$.

2) $\Gamma(t ; 0,0,0)=C(t)$.

3) There is $\delta>0$ and a real $K$ such that

$$
\delta<\left|\operatorname{Det}\left(\gamma_{\alpha \beta}\right)\right|, \quad\left|\gamma_{\alpha \beta}\right|<K, \quad\left|a_{\alpha}\right|<K, \quad\left|\omega_{\alpha \beta}\right|<K, \quad\left|\tilde{K}_{\alpha \beta}\right|<K^{3}
$$

for every $\alpha, \beta=1,2,3$ and $0<t<2,\left(u^{1}\right)^{2}+\left(u^{2}\right)^{2}+\left(u^{3}\right)^{2}<\varepsilon^{2}$; all functions $\gamma_{\alpha \beta}, a_{\alpha}, \omega_{\alpha \beta}, \tilde{K}_{\alpha \beta}$ are continuous for $\alpha, \beta=1,2,3$.

Denote by $X$ the component of $Z$ containing $C([0,1))$. Without any loss of generality, we may assume:

i) $C((1,2]) \subset U-\bar{X}^{U}$. If not, then there certainly is a curve, $C^{\prime}$, say, of $\Gamma$ having a non-empty intersection with $X$ and $U-\bar{X}^{U}$, thus we can choose $C^{\prime}$ instead of $C$, introduce a proper parameter on it and find $\varepsilon$.

\footnotetext{
${ }^{2} C$ must be one-to-one, because it is time-like and causality holds throughout $U$.

${ }^{3}$ Here $\gamma_{\alpha \beta}$ is the standard spatial metric, $a_{\alpha}$ is the curvature vector, $\omega_{\alpha \beta}$ is the space vortex tensor and $\tilde{K}_{\alpha \beta}$ is the Born tensor of the congruence $\Gamma$, see, e.g. $[13,14]$. These magnitudes behave like scalars under the transformation of $x^{i}$ and like tensors under those of $u^{\alpha}$.
} 
ii) The closure in $U$ of the set of all points $\Gamma\left(0 ; u^{1}, u^{2}, u^{3}\right)$, when $\left(u^{1}\right)^{2}+\left(u^{2}\right)^{2}+\left(u^{3}\right)^{2}<\varepsilon^{2}$, lies in $X$ and every curve of $\Gamma$ cuts $\dot{X}^{U}$ just once (it is sufficient to choose $\varepsilon$ properly and introduce a new parameter, if this is not the case).

iii) There is a point $p^{\prime} \in V$ such that $p^{\prime} Y p$. If not, then there are points of $U$ in every neighbourhood of $p$, for which such points of $V$ must exist (see Theorem 2), and we had only to choose another curve of $\Gamma$ instead of $C$.

iv) There is a sequence of points $p_{i} \in X$ converging to both $p$ and $p^{\prime}$ : We know that there certainly is a sequence of points in $Z$ with this property. If only a finite number of points of every such sequence lies in $X$, then $Z$ must have one more component, $X_{1}$, say, such that $p$ lies on $\dot{X}_{1}^{U}$. There cannot be more than two such components, or else their common boundary in $p$ could not be a hypersurface. Then, every sequence $\left\{p_{i}\right\}$ has an infinite number of points in $X_{1}$ and we can perform our proof with $X_{1}$ instead of $X$.

Now, let us introduce the parameter $s$ in place of $t$ such that $s \geqq 0$, $s=0$ only if $t=0$, and $s$ measures the proper time along each curve of $\Gamma$. As each curve of $\Gamma$ cuts $\dot{X}^{U}$ just once and $\dot{X}^{U}$ is a hypersurface, we have on $\dot{X}^{U} s=f\left(u^{1}, u^{2}, u^{3}\right)$, where $f$ is a continuous function.

We choose a normal coordinate neighbourhood $V_{p^{\prime}}$ of $p^{\prime}$ in $V$ that is simultaneously a local causality neighbourhood (see, e.g. [1]); denote the coordinates $y^{0}, y^{1}, y^{2}, y^{3}\left(y^{0}\left(p^{\prime}\right)=y^{1}\left(p^{\prime}\right)=y^{2}\left(p^{\prime}\right)=y^{3}\left(p^{\prime}\right)=0\right)$. There is a coordinate ball $B$ defined by $\left(y^{0}\right)^{2}+\left(y^{1}\right)^{2}+\left(y^{2}\right)^{2}+\left(y^{3}\right)^{2}<r^{2}$ such that $\bar{B}^{V}$ is compact, lies in $V_{p^{\prime}}$, and has no point in common with $\Gamma\left(0 ; u^{1}, \mathrm{u}^{2}, u^{3}\right)$ for all $\left(u^{1}\right)^{2}+\left(u^{2}\right)^{2}+\left(u^{3}\right)^{2}<\varepsilon^{2}$ and with $\{C \mid X\}$, where $C \mid X$ denotes that part of the curve $C$ lying in $X$. Such a choice of $B$ is always possible, because $C \mid X$ has no limit point in $V-Z$, or else $V$ would not be strongly causal according to the Lemmas 1,2 , and 3 .

There is $N$ such that $p_{i} \in B$ and simultaneously lies on some curve of $\Gamma, C_{i}$, say, for every $i>N$. There must be a point, denote it by $q_{i}$, where the curve $C_{i}$ cuts $\dot{B}^{V}$ for the first time, if $i>N$. Next, every $C_{i} \mid X$ must leave $\bar{B}^{V}$ : if all points of $C_{i} \mid X$ for $s>s\left(q_{i}\right)$ lie in $\bar{B}^{V}$, then there would have to be at least one limit point of $C_{i} \mid X$ in $\bar{B}^{V}-X$, because $\bar{B}^{V}$ is compact and the set $C_{i}\left(\left[s\left(q_{i}\right), f\left(u^{1}\left(q_{i}\right), u^{2}\left(q_{i}\right), u^{3}\left(q_{i}\right)\right)\right)\right)$ is closed in $X$ but not compact; applying the Lemmas 1,2, and 3 , we should not find, then, $V$ to be strongly causal. Thus, every $C_{i} \mid X$ has a second intersection with $\dot{B}^{V}$, say, $r_{i} . r_{i}$ is the last intersection of $C_{i} \mid X$ with $\bar{B}^{V}$, because $V_{q}$ is a local causality neighbourhood. Choose a subsequence $\left\{C_{i}^{\prime} \mid X\right\}$ such that the corresponding subsequences $\left\{q_{i}^{\prime}\right\}$ and $\left\{r_{i}^{\prime}\right\}$ converge to some points $q$ and $r$ on $\dot{B}^{V}$ (in fact, we have $\left\{C_{i}^{\prime} \mid X\right\}=\left\{C_{i} \mid X\right\}$, see below). It holds

$$
f\left(u^{1}\left(p_{i}^{\prime}\right), u^{2}\left(p_{i}^{\prime}\right), u^{3}\left(p_{i}^{\prime}\right)\right)>s\left(r_{i}^{\prime}\right)>s\left(p_{i}^{\prime}\right)>s\left(q_{i}\right),
$$


therefore

$$
f(0,0,0) \geqq \lim _{i \rightarrow \infty} s\left(r_{i}^{\prime}\right) \geqq \lim _{i \rightarrow \infty} s\left(p_{i}^{\prime}\right) \geqq \lim _{i \rightarrow \infty} s\left(q_{i}^{\prime}\right) .
$$

But $\lim _{i \rightarrow \infty} s\left(q_{i}^{\prime}\right)<f(0,0,0)$ is impossible, or else the point $r$ lies on $C \mid X$, contrary to the choice of $B$. Hence

$$
\lim _{i \rightarrow \infty} s\left(r_{i}^{\prime}\right)=\lim _{i \rightarrow \infty} s\left(p_{i}^{\prime}\right)=\lim _{i \rightarrow \infty} s\left(q_{i}^{\prime}\right)=f(0,0,0) .
$$

We denote the set of all points lying on the curves of $\Gamma$ by $\{\Gamma\}$. The set $\{\Gamma\} \cap B$ is open and the four functions $y^{0}, u^{1}, u^{2}, u^{3}$ are well defined and differentiable on it. They can be chosen as coordinates there: the sets $y^{0}=$ Const. are three-dimensional smooth space-like hypersurfaces, the curves $u^{1}=$ Const., $u^{2}=$ Const., $u^{3}=$ Const. form a regular threeparameter time-like congruence and each of them intersects each hypersurface $y^{0}=$ Const. only once, because it goes only once through $V_{p^{\prime}}$. The boundary of the set $\{\Gamma\} \cap B$ in $V$ consists of two disjoint sets $\{\Gamma\} \cap \dot{B}^{V}$ and $\left(\overline{\{\Gamma\}^{V}}-\{\Gamma\}\right) \cap \bar{B}^{V}$. The coordinate system $\left(y^{0}, u^{\alpha}\right)$ can easily be extended to the points of $\{\Gamma\} \cap \dot{B}^{V}$. Let $\bar{q} \in\left(\{\bar{\Gamma}\}^{V}-\{\Gamma\}\right) \cap \bar{B}^{V}$ and $\left\{\bar{q}_{i}\right\}$ a sequence of points of $\{\Gamma\} \cap B$ converging to $\bar{q}$. Let the curve of $\Gamma$ going through $\bar{q}_{i}$ be denoted by $\bar{C}_{i}$. From the conditions 3$)_{1,2,5}$ it follows that the curves $\bar{C}_{i} \mid \bar{B}^{V}$ converge to a curve $\bar{\lambda}$ going through $\bar{q}$. Thus, if $\left\{\bar{q}_{i}^{\prime}\right\}$ is another point sequence in $\{\Gamma\} \cap B$ converging to $\bar{q}$, then the corresponding limit curve is identical to $\bar{\lambda}$ : the sequence $\left\{\bar{q}_{i}^{\prime \prime}\right\}$ defined by $\bar{q}_{2 k}^{\prime \prime}=\bar{q}_{k}, \bar{q}_{2 k-1}^{\prime \prime}=\bar{q}_{k}^{\prime}$ must give a curve. The curve $\bar{\lambda}$ is smooth in view of 3$)_{3,4}$ and as a limit curve of a sequence of time-like curves is causal. It intersects, therefore, each hypersurface $y^{0}=$ Const. at most once, and we can extend the coordinate system $\left(y^{0}, u^{\alpha}\right)$ also in the points of $\left(\{\bar{\Gamma}\}^{V}-\{\Gamma\}\right) \cap \bar{B}^{V}$.

Let $g_{i j}$ be the components of the metric tensor in the coordinates $\left(y^{0}, u^{\alpha}\right)$. According to $(2) g_{00}(0,0,0,0)=0$ must hold. Further, $g_{0 \alpha}(0,0,0,0)$ is bounded for all $\alpha$, non-null for at least one $\alpha$, and $g_{\alpha \beta}(0,0,0,0)$ is bounded for all $\alpha, \beta$, or else the system $\left(y^{0}, u^{\alpha}\right)$ would not be regular in $p^{\prime}$. The functions $g_{i j}\left(y^{0}, u^{\alpha}\right)$ are continuous in $\overline{\{\Gamma\} \cap B^{V}}$. As $\left(y^{0}, u^{\alpha}\right)$ are co-moving coordinates (see, e.g., [14]), we have

and this implies

$$
\gamma_{\alpha \beta}=g_{\alpha \beta}-\frac{g_{\alpha 0} g_{\beta 0}}{g_{00}}
$$

contrary to 3$)_{2}$.

$$
\left|\lim _{\substack{u^{1} \rightarrow 0 \\ u^{2} \rightarrow 0 \\ u^{3} \rightarrow 0}} \gamma_{\alpha \beta}\left(0, u^{1}, u^{2}, u^{3}\right)\right|=+\infty,
$$

Acknowledgment. The present author wishes to express his gratitude to Prof. Dr. A. Mercier for his encouraging and supporting this research. The first draft of this paper was read and commented by Dr. S. W. Hawking. It is a pleasant duty to thank for this comment, which was used to correct some points. Thanks go to Dr. W. Kundt for many improvements in the manuscript. 


\title{
References
}

1. Hawking, S. W.: Proc. Roy. Soc. (London), Ser. A 300, 187 (1967).

2. - Penrose, R.: Proc. Roy. Soc. (London), Ser. A314, 529 (1970).

3. - Ellis, G.F.R.: Astrophys. J. 152, 25 (1968).

4. Carter, B.: Phys. Rev. 174, 1559 (1968).

5. Israel, W.: Phys. Rev. D2, 641 (1970).

6. Geroch, R.: J. Math. Phys. 9, 450 (1968).

7. Schmidt, B.G.: Contribution. to Seminar on Bearing of Topology upon General Relativity, Gwatt, Switzerland, May 1970.

8. Hajicek, P.: Commun. math. Phys. 17, 109 (1970).

9. - J. Math. Phys., to be published.

10. Hicks, N. J. : Notes on Differential Geometry. Princeton: Van Nostrand 1965.

11. Hocking, J. G., Young, G. S. : Topology. Reading: Addison-Wesley 1961.

12. Geroch, R.: Unpublished.

13. Cattaneo, C.: Nuovo Cimento XIII, 237 (1959).

14. Boyer, R. H.: Proc. Roy. Soc. (London), Ser. A 283, 343 (1963).

\author{
P. Hajicek \\ Universität Bern \\ Institut $\mathrm{f}$. Theoretische Physik \\ CH 3000 Bern, Sidlerstr. 5
}

